\title{
Inhibition of Tet1- and Tet2-mediated DNA demethylation promotes immunomodulation of periodontal ligament stem cells
}

\author{
Tingting Yu $\mathbb{0}^{1,2}$, Dawei Liu' ${ }^{1,2}$, Ting Zhang ${ }^{1}$, Yanheng Zhou' ${ }^{1}$, Songtao Shi ${ }^{2,3}$ and Ruili Yang ${ }^{1,2}$
}

\begin{abstract}
Periodontal ligament stem cells (PDLSCs) possess great potential for clinical treatment of immune diseases due to their extensive immunomodulatory properties. However, the underlying mechanisms that govern the immunomodulatory properties of mesenchymal stem cells (MSCs) are still not fully elucidated. Here, we show that member of the Teneleven translocation (Tet) family, a group of DNA demethylases, are capable of regulating PDLSC immunomodulatory functions. Tet 1 and Tet2 deficiency enhance PDLSC-induced T cell apoptosis and ameliorate the disease phenotype in colitis mice. Mechanistically, we found that downregulation of Tet1 and Tet2 leads to hypermethylation of DKK-1 promoter, leading to the activation of WNT signaling pathway and therefore promoting Fas ligand (FasL) expression, which results in elevated immunomodulatory capacity of PDLSCs. These results reveal a previously unrecognized role of Tet1 and Tet2 in regulating immunomodulation of PDLSCs. This Tet/DKK-1/FasL cascade may serve as a promising target for enhancing PDLSC-based immune therapy.
\end{abstract}

\section{Introduction}

The Ten-eleven translocation (Tet) family is a group of recently identified demethylases capable of modifying DNA by hydroxylating 5-methylcytosine $(5-\mathrm{mC})$ to 5 hydroxymethylcytosine $(5-\mathrm{hmC})^{1-3}$. This discovery revealed a novel mechanism by which Tet enzymes regulate DNA demethylation. Three Tet family members (Tet1, Tet2, and Tet3) show distinct expression patterns depending on cell or tissue type and developmental stage $^{4,5}$. For instance, Tet1 is highly expressed in mouse embryonic stem cells (ESCs); upon ESC differentiation, its expression level is significantly decreased. However, some terminally differentiated cells, such as Purkinje neurons, also show upregulated Tet1 expression ${ }^{2,6,7}$. Emerging evidences indicate that Tet-mediated DNA demethylation

\footnotetext{
Correspondence: Ruili Yang (ruiliyangabc@163.com)

${ }^{1}$ Department of Orthodontics, Peking University School and Hospital of Stomatology, \#22 Zhongguancun South Avenue, 100081 Beijing, China ${ }^{2}$ Department of Anatomy and Cell Biology, School of Dental Medicine, University of Pennsylvania, Philadelphia, PA 19104, USA Full list of author information is available at the end of the article. Edited by $Y$. Shi
}

contributes to normal growth and developmental processes as well as disease development. Alteration of Tet expression has been linked to tumorigenesis, neural disorders, and $\mathrm{T}$ cell-related immune disorders ${ }^{8}$. Our previous studies showed that Tet 1 and Tet2 play a crucial role in maintaining bone marrow mesenchymal stem cell (BMMSC) functions and bone homeostasis. Tet1 and Tet2 depletion resulted in osteopenia and impairment of BMMSC differentiation ${ }^{9}$, suggesting that the Tet family is able to modulate MSC biological function. Moreover, we have found that Tet 1 and Tet2 help to maintain immune homeostasis via regulating regulatory $\mathrm{T}$ (Treg) cell function ${ }^{10}$. Whether Tet1/Tet2-mediated DNA demethylation modulates MSC-mediated immunomodulation remains unclear.

Periodontal ligament stem cells (PDLSCs), isolated from periodontal ligament tissue, possess unique immunomodulatory capacity ${ }^{11}$. PDLSCs interact with different immune cells, for example, suppressing $\mathrm{T}$ cell proliferation $^{12}$ and upregulating Tregs ${ }^{13}$. After transplantation in a periodontitis minipig model, PDLSCs were able to

\section{(c) The Author(s) 2019}

(c) (i) Open Access This article is licensed under a Creative Commons Attribution 4.0 International License, which permits use, sharing, adaptation, distribution and reproduction cc) in any medium or format, as long as you give appropriate credit to the original author(s) and the source, provide a link to the Creative Commons license, and indicate if changes were made. The images or other third party material in this article are included in the article's Creative Commons license, unless indicated otherwise in a credit line to the material. If material is not included in the article's Creative Commons license and your intended use is not permitted by statutory regulation or exceeds the permitted use, you will need to obtain permission directly from the copyright holder. To view a copy of this license, visit http://creativecommons.org/licenses/by/4.0/. 
modulate the local immune microenvironment and result in periodontal tissue regeneration ${ }^{14}$. Therefore, it is possible to use PDLSCs to treat inflammatory diseases ${ }^{14,15}$, such as periodontal disease.

In the present study, we demonstrated that Tet1/Tet2mediated DNA demethylation is capable of regulating the immunomodulatory function of PDLSCs. Tet1 and Tet2 deficiency enhances PDLSC-induced T cell apoptosis and ameliorates the disease phenotype in colitis mice.

\section{Results}

\section{Human PDLSCs express Tet 1 and Tet2}

We found that human PDLSCs (hPDLSCs) isolated from human periodontal ligament expressed elevated levels of Tet1 and Tet2, as assessed by Western blotting and real-time polymerase chain reaction (qPCR), when compared to human BMMSCs (hBMMSCs) (Fig. 1a, b). Immunostaining showed that MSC marker CD146 was co-expressed with Tet1 and Tet2 in hPDLSCs (Fig. 1c). These data indicate that PDLSCs express certain levels of Tet1 and Tet2.

\section{Tet1 and Tet2 regulate PDLSC-mediated immunomodulation}

To investigate whether Tet1 and Tet2 mediate DNA demethylation that may regulate the multi-lineage differentiation and immunomodulation capacities of
PDLSCs, we knocked down Tet 1 and Tet2 expression in PDLSCs by using small interfering RNAs (siRNAs) (Fig. 2a). Bromodeoxyuridine (BrdU)-labeling assays showed that knocking down Tet1 and Tet2 in PDLSCs led to upregulated proliferation when compared with the control group (Fig. 2b). Flow cytometric analysis showed that MSC surface markers, including CD105 and CD73, were significantly elevated in Tet1/Tet2 siRNA-treated PDLSCs compared with control PDLSCs, but not CD90. The hematopoietic lineage markers, CD34 and CD45, were absent in Tet $1 / 2$ siRNA-treated PDLSCs, similar to the observations of control PDLSCs (Fig. 2c). Furthermore, we cultured PDLSCs and Tet1/Tet2 siRNA-treated PDLSCs under osteogenic and adipogenic differentiation condition and found that Tet 1 and Tet 2 deficiency led to significantly decreased osteogenic (Fig. S1a, b) and adipogenic (Fig. S1c, d) differentiation potential when compared to the control PDLSCs.

Next, to evaluate the immunomodulatory properties of PDLSCs, we co-cultured PDLSCs with T cells and found that PDLSCs were capable of inducing $\mathrm{T}$ cell apoptosis ${ }^{16}$. Moreover, Tet1/Tet2 siRNA-treated PDLSCs had a significantly elevated capacity to induce AnnexinV ${ }^{+} 7 \mathrm{AAD}^{+}$ double-positive $\mathrm{T}$ cell apoptosis, when compared to the control PDLSCs (Fig. 2d, e). These results indicate that the inhibition of Tet1 and Tet2 promotes PDLSCs' immunomodulatory capacity by inducing $\mathrm{T}$ cell apoptosis.
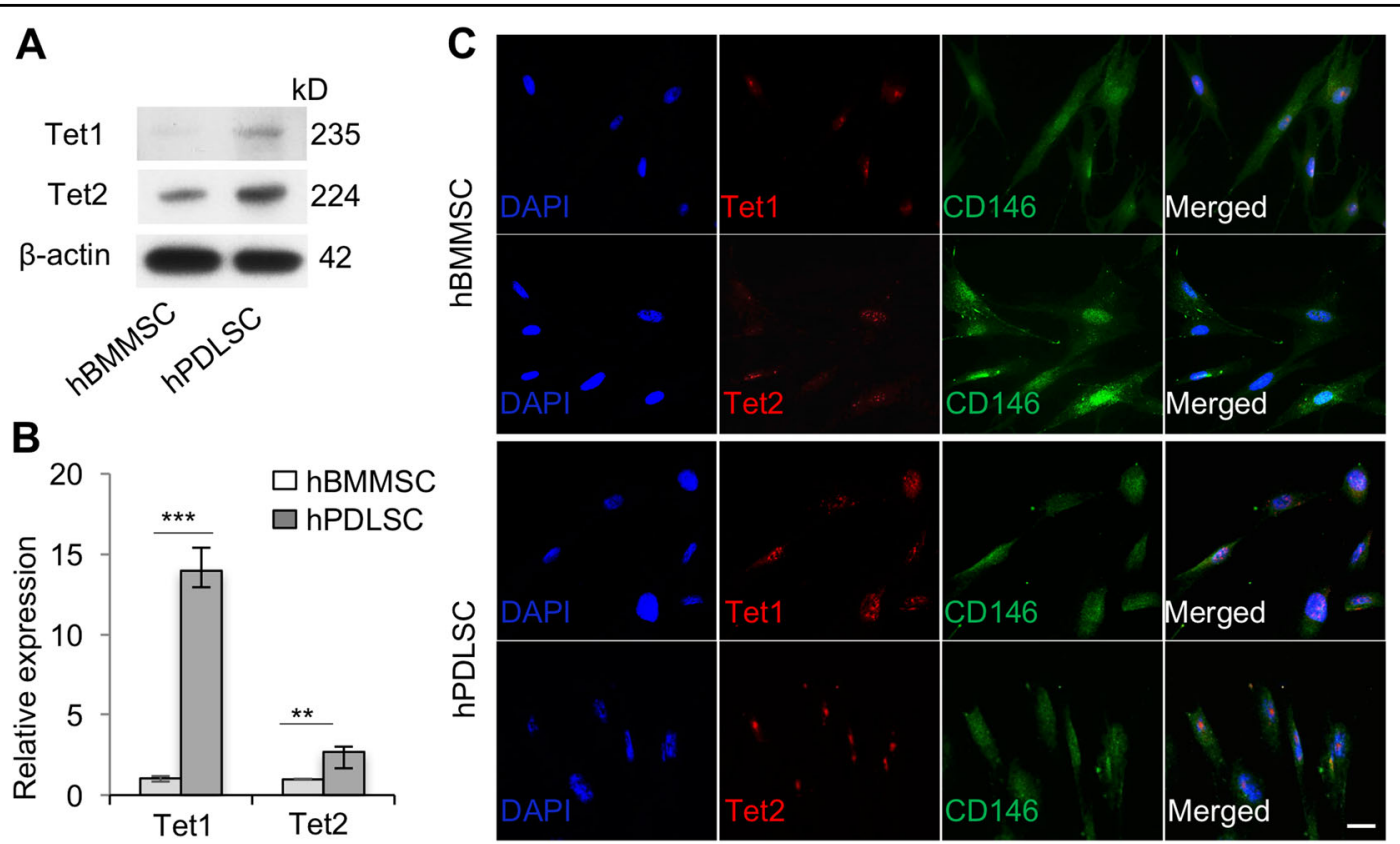

Fig. 1 Human periodontal ligament stem cells (hPDLSCs) express Tet1 and Tet2. a, b Both human bone marrow mesenchymal stem cells (hBMMSCs) and hPDLSCs expressed Tet1 and Tet2, as assessed by Western blotting (a) and QPCR (b). c Immunocytofluorescent staining showed that CD146-positive hPDLSCs express Tet1 and Tet2. Scale bar, $50 \mu \mathrm{m} .{ }^{* * *} P<0.001,{ }^{* *} p<0.01 ; p$ values were calculated using two-tailed Student's $t$ test $($ mean \pm SD) 


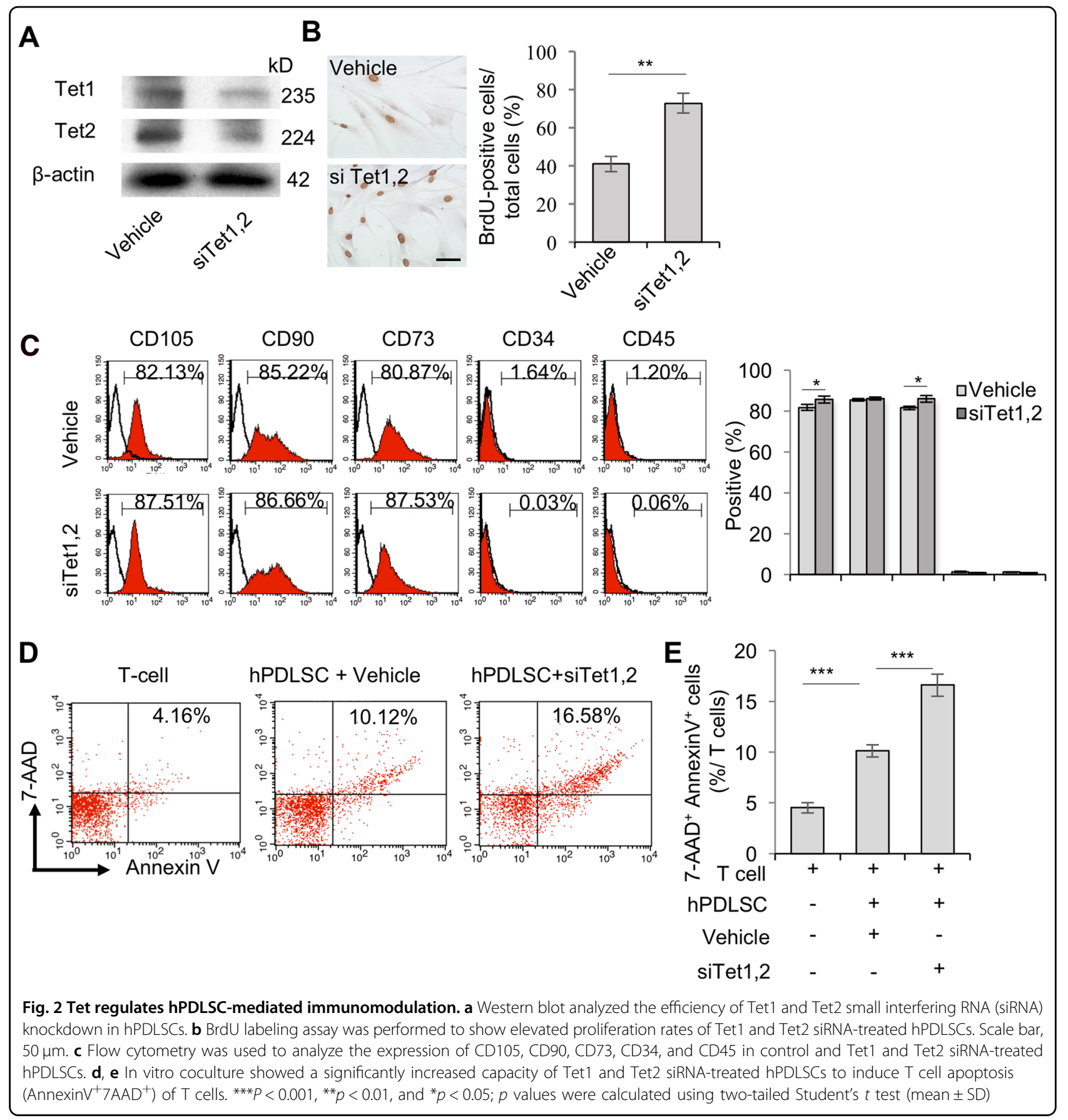

Inhibition of Tet 1 and Tet 2 enhances therapeutic effect of PDLSCs in treating colitis

The ability of MSCs to modulate immune response is one of their most important characteristics ${ }^{17,18}$. To further assess the role of Tet 1 and Tet 2 in immunomodulation by PDLSCs, we compared the immunotherapeutic effects of control and Tet1/Tet2 siRNA-treated PDLSCs in experimental colitis mice. C57BL/6J mice were orally administered 3\% dextran sodium sulfate (DSS) for 10 days to establish acute colitis. On day 3 , colitis mice were treated with Tet1/Tet2 siRNA-treated PDLSCs or control PDLSCs by systemic transplantation through tail vein, followed by sacrifice of the mice on day 10 to collect samples for evaluation (Fig. 3a).

Consistent with previous reports ${ }^{19}$, DSS-induced colitis mice lost weight at a sustained rate and exhibited bloody diarrhea/loose feces, which were characterized by an overall evaluation of their condition using the established disease activity index (DAI). Infusion of either Tet1/Tet2 siRNA-treated PDLSCs or control PDLSCs partially 


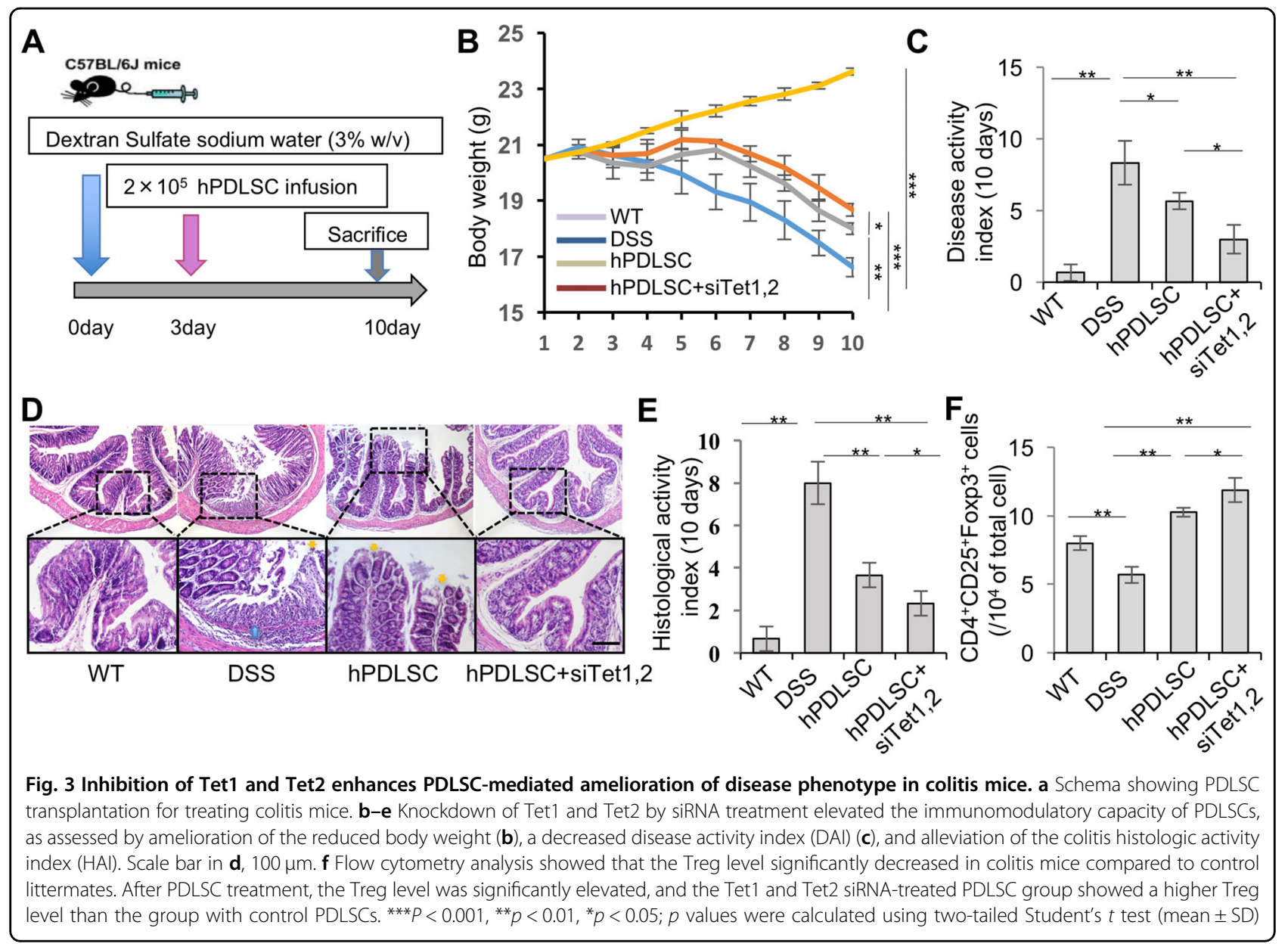

restored the reduced body weight of the colitis mice and decreased their DAI scores. Furthermore, Tet1/ Tet2 siRNA pretreatment was able to enhance the ability of PDLSCs to restore the reduced body weight (Fig. 3b) and decreased the DAI scores (Fig. 3c). Histologically, localized inflammatory cell infiltration, epithelial ulceration, and impairment of crypt architecture were observed in the intestines of DSS-induced colitis mice. Systemic infusion of either Tet1/Tet2 siRNA-treated PDLSCs or control PDLSCs could recover impaired intestinal structure. However, Tet1/Tet2 siRNA-treated PDLSCs demonstrated a superior ability to eliminate the inflammatory cells and recover the epithelial structure, as assessed by the histologic activity index (HAI) (Fig. 3d, e). Moreover, decreased number of $\mathrm{CD} 4{ }^{+} \mathrm{CD} 25^{+} \mathrm{Foxp} 3^{+}$ Tregs were observed in the mice with colitis at day 10 post-DSS induction (Fig. 3f). Infusion of control PDLSCs elevated the number of Treg cells, and Tet1/Tet2 siRNA pretreatment enhanced this effect. Taken together, these experimental data indicate that Tet 1 and Tet 2 inhibition results in an elevated capacity for PDLSC-mediated immunomodulation.

\section{Tet1 and Tet2 regulate FasL expression through Wnt/ $\beta$-catenin pathway in PDLSCs}

To explore the underlying mechanism of how Tet1 and Tet2 regulate immunomodulation of PDLSCs, we examined the levels of FasL, a key member of the apoptosis pathway, in PDLSCs. It has been reported that FasL is related to MSCs' immunomodulatory ability, as a previous study demonstrated that FasL in MSCs induced $\mathrm{T}$ cell apoptosis and immune tolerance ${ }^{20}$. Thus, in order to confirm that PDLSCs express FasL, we used double immunostaining and found that CD146-positive PDLSCs expressed FasL (Fig. 4a). To investigate whether Tet1 and Tet2 modulate the FasL expression level, Western blot and qPCR analysis were used, and it revealed that FasL was significantly elevated in Tet $1 /$ Tet 2 siRNA-treated PDLSCs (Fig. 4b, c), indicating that the inhibition of Tet1 and Tet2 upregulates FasL expression and results in an elevation of MSC-based immunomodulatory ability. Next, to evaluate whether FasL is a direct target of Tet1 and Tet2, we used the Methprimer software to detect the promoter of FasL and found that the FasL promoter lacks CpG island, which indicates that Tet1 and Tet2 may not 


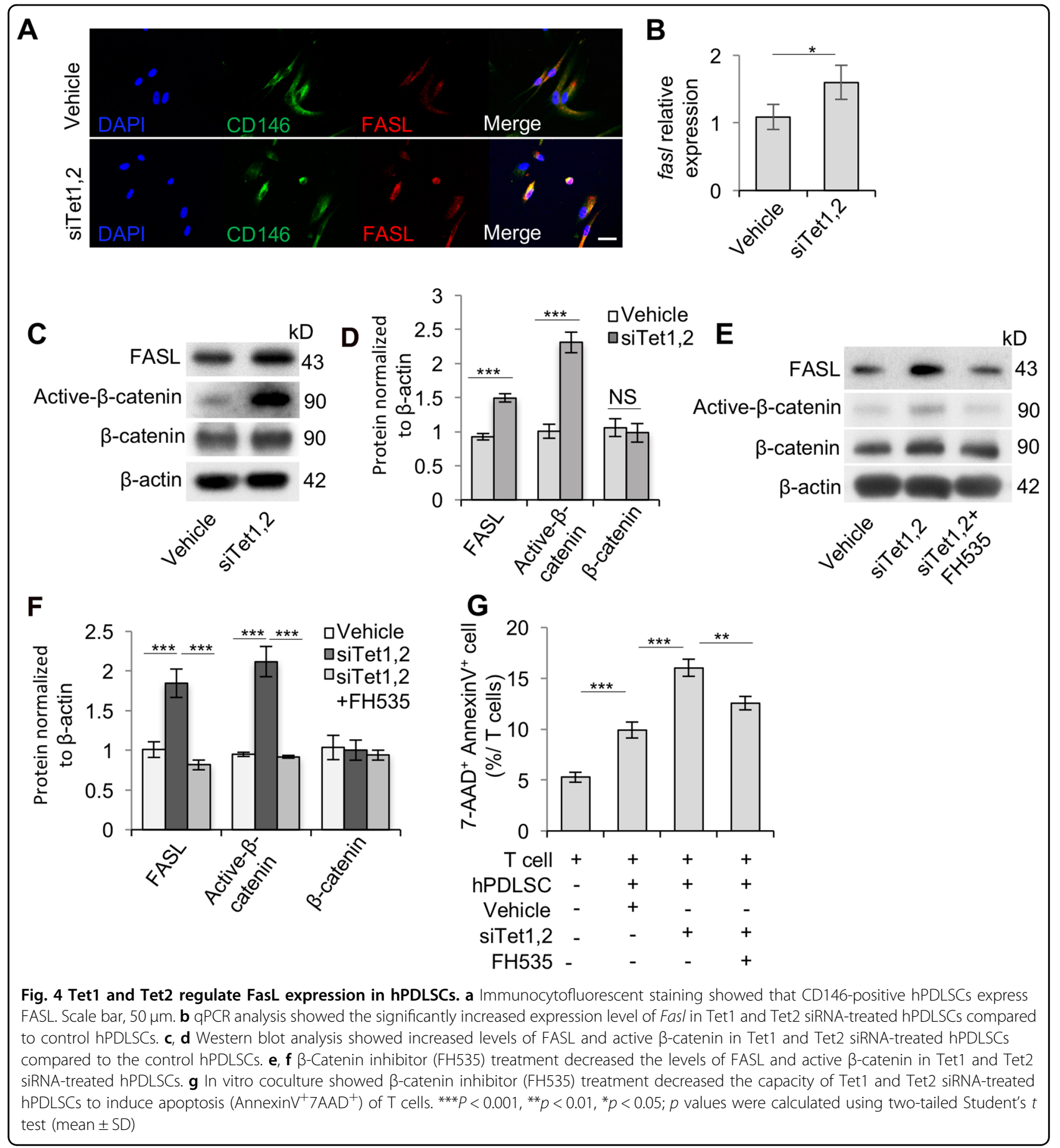

prefer to bind to the promoter of FasL ${ }^{21,22}$. Thus, we continued searching for potential molecules that may connect Tet with FasL. Since $\beta$-catenin could serve as a transcriptional factor to regulate FasL expression ${ }^{23,24}$, we next examined the $\mathrm{Wnt} / \beta$-catenin expression levels in Tet1/Tet2 siRNA-treated PDLSCs. Western blotting showed that the expression level of active $\beta$-catenin (nonphosphorylated) was indeed significantly elevated, although the total level of $\beta$-catenin was not significantly elevated in Tet1/Tet2 siRNA-treated PDLSCs (Fig. 4c, d). The expression levels of active $\beta$-catenin and FasL were significantly decreased after the treatment of $\beta$-catenin inhibitor (FH535) (Fig. 4e, f). When co-cultured with $\mathrm{T}$ cells, Tet1/Tet2 siRNA-treated PDLSCs gained an elevated capacity to induce AnnexinV ${ }^{+} 7 \mathrm{AAD}^{+}$doublepositive $\mathrm{T}$ cell apoptosis when compared to the 
untreated group, but the elevated induction of apoptosis could be abrogated by $\beta$-catenin inhibitor (FH535) treatment (Fig. 4g). These results suggest that Tet1 and Tet2 act as upstream regulators of $\mathrm{Wnt} / \beta$-catenin signaling to modulate FasL expression, which in turn regulates the PDLSC-mediated immunomodulatory capacity.

Furthermore, besides FasL, many other target genes can be regulated by $\beta$-catenin. Therefore, we analyzed Notch and tumor growth factor- $\beta$ signaling-related genes, which have been identified as the downstream of WNT/ $\beta$-catenin pathway ${ }^{25,26}$. Western blot analysis showed that the expression level of P-smad3 was decreased, while Notch1 and Notch2 showed an elevated expression in Tet1/Tet2 siRNA-treated PDLSCs compared with the control group (Fig. S2). These results indicated that Tet might be able to regulate other WNT-associated genes.

\section{Tet1 and Tet2 serve as demethylases on DKK-1 promoter to regulate FasL expression in PDLSCs}

To investigate how Tet1/Tet2 regulate Wnt/ $\beta$-catenin signaling, we examined the level of DKK-1, an upstream inhibitor of the WNT pathway ${ }^{27}$, and found that DKK-1 was downregulated in Tet1/Tet2 siRNA-treated PDLSCs. Immunofluorescence staining showed co-localization of DKK-1 with MSC surface marker CD146 (Fig. 5a). Western blotting (Fig. 5b) and qPCR (Fig. 5c) showed that the expression level of DKK- 1 was significantly decreased in Tet1/Tet2 siRNA-treated PDLSCs compared to the control group. As previous studies indicated that Tet1 and 5$\mathrm{hmC}$ prefer to co-localize at CpG-rich locus in the promoter transcriptional start $\operatorname{sites}^{21,22}$, we examined the promoter region of DKK-1 (DKK-1 -1500 to 0, Homo sapiens chromosome 10, GRCh38 52,312,781 to $52,314,281$ ) by using the Methprimer software; three CpG islands (DKK-1 -1048 to $-943,-915$ to $-804,-286$ to -176 ) were detected (Fig. 5d). To demonstrate if Tet1 and Tet2 are able to regulate $D K K-1$ directly, chromatin immunoprecipitation-qPCR (ChIP-qPCR) analysis were used to verify that Tet 1 and Tet 2 were able to bind to the CpG islands of the DKK-1 promoter (Fig. 5e). We next examined whether inhibition of Tet 1 and Tet 2 affected the enrichment of 5-hmC level at the DKK-1 promoter. Hydroxymethylated DNA immunoprecipitation-qPCR (hMeDIP-qPCR) analysis showed that Tet1/Tet2 siRNAtreated PDLSCs displayed a significantly decreased 5-hmC level compared to control PDLSCs on the three CpG island sites of the $D K K-1$ promoter (Fig. $5 \mathrm{f}$ ). Oxidative bisulfite-sequencing (OxBS) sequencing analysis also showed an elevated methylation status in the promoter of DKK-1 locus in Tet1/Tet2 siRNA-treated PDLSCs compared to control PDLSCs (Fig. 5g). These results demonstrated that Tet1 and Tet2 are able to directly regulate $D K K-1$ expression through DNA demethylation. Next, we added DKK-1 to Tet1/Tet2 siRNA-treated
PDLSCs and found that exogenous DKK-1 could block the increased expression of active $\beta$-catenin and FasL that was induced by Tet1/Tet2 siRNA treatment (Fig. 5h). When co-cultured with $\mathrm{T}$ cells, Tet1/Tet2 siRNA-treated PDLSCs gained an elevated capacity to induce AnnexinV $^{+} 7 \mathrm{AAD}^{+}$double-positive $\mathrm{T}$ cell apoptosis when compared to the untreated group, but that effect could be abrogated by DKK-1 treatment (Fig. 5i). Taken together, these findings indicate that the inhibition of Tet 1 and Tet2 could promote PDLSC-mediated immunomodulation ability through regulating $D K K-1$ demethylation (Fig. 6).

\section{Discussion}

Tet-mediated conversion of 5-mC to 5 -hmC has been proposed as the initial step in active DNA demethylation, which plays an important role in maintaining tissue homeostasis ${ }^{10,28,29}$. Inhibition of Tet leads to deficiency of Tregs $^{28}$ and myeloid invariant natural killer T cells, as well as B cell malignancy ${ }^{30-32}$. Our previous study demonstrated that Tet 1 and Tet 2 are required to maintain bone homeostasis via regulating the osteogenic differentiation of BMMSCs ${ }^{9,33}$. Here, we showed that inhibition of Tet1 and Tet2 led to decreased osteogenic and adipogenic differentiation of PDLSCs (Fig. S1). Moreover, we showed that Tet 1 and Tet 2 deficiency promoted the proliferation of hPDLSCs (Fig. 2b), in agreement with our previous finding in mouse BMMSCs ${ }^{9}$. However, based on studies on umbilical cord (UC)-derived MSCs, loss of Tet1/2 can inhibit the ability of MSCs to replicate ${ }^{34}$, which implies that different effects of Tet $1 / 2$ on MSC proliferation may be regulated by specific molecules ${ }^{9,34,35}$.

MSCs possess great potential for treating immune diseases due to their extensive immunomodulatory properties $^{16,20}$. Whether Tet participates in regulating MSC immunomodulation has remained unknown. In the present study, we demonstrated that inhibition of Tet1 and Tet2 promoted PDLSC-induced T cell apoptosis (Fig. 2d, e). Importantly, when PDLSCs were systematically infused into DSS-induced colitis mice, Tet1/Tet2-downregulated PDLSCs showed significantly increased therapeutic effects (Fig. 3). These results may provide a promising method to promote MSC-mediated therapeutic efficacy.

The multi-potent and immunomodulatory properties of MSCs make them a valuable cell source for regeneration and immune therapy ${ }^{36,37}$. The key issue in MSC biology is to illustrate the underlying mechanisms that control MSCs' multiple distinct functions. In the present study, we demonstrated that Tet deficiency led to inhibition of PDLSC differentiation toward osteogenic and adipogenic lineages (Fig. S1), similar to the observations made concerning BMMSCs in our previous report ${ }^{9}$. Interestingly, we also found that inhibition of Tet1 and Tet2 could elevate PDLSC immunomodulatory potential (Figs. 2 and 3), which indicates that Tet 1 and Tet 2 might serve as switch 


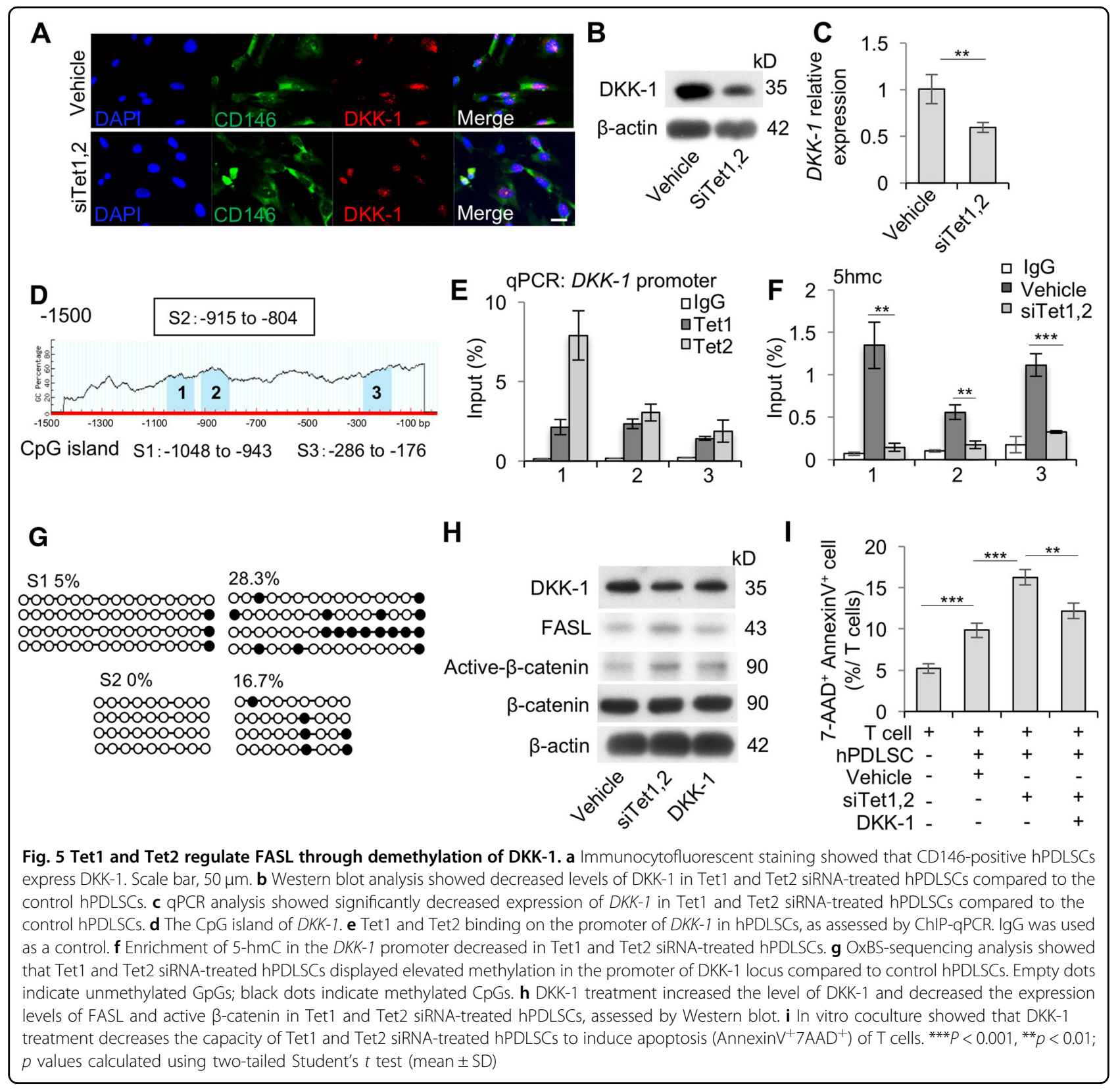

genes to control MSC differentiation and immunomodulation. Downregulation of Tet1 and Tet2 resulted in differentiation deficiency as well as upregulation of immunomodulation ability in PDLSCs. However, based on our current understanding, more investigation is required to further illustrate the mechanisms of how Tet regulates MSCs' distinct functions in differentiation and immunomodulation.

Mechanistically, we identified a novel role of Tet1 and Tet2 in binding directly to the $D K K-1$ promoters, and further identified that they act as an upstream regulator of Wnt/ $\beta$-catenin pathway to modulate FasL expression (Fig. 6). Previous studies demonstrated that Wnt pathway inhibitor DKKs could be inactivated by DNA methylation in cancer cells to sustain tumor development ${ }^{38,39}$, which can be reversed by demethylation activity on the $D K K$ promoter region ${ }^{40}$. In the present work, we reported that Tet1 and Tet2 bind to the promoter of $D K K-1$ to maintain its hypomethylation in PDLSCs. It is known that MSCs are able to induce $\mathrm{T}$ cell apoptosis via Fas/FasL pathway, resulting in upregulation of Tregs and immune tolerance $^{20}$. $\beta$-Catenin could directly bind to the FasL promoter to drive gene expression at the transcriptional level $^{23}$. Based on our findings, downregulation of Tet1 and Tet2 led to repression of $D K K-1$ by DNA methylation modification, which results in activation of Wnt pathway 


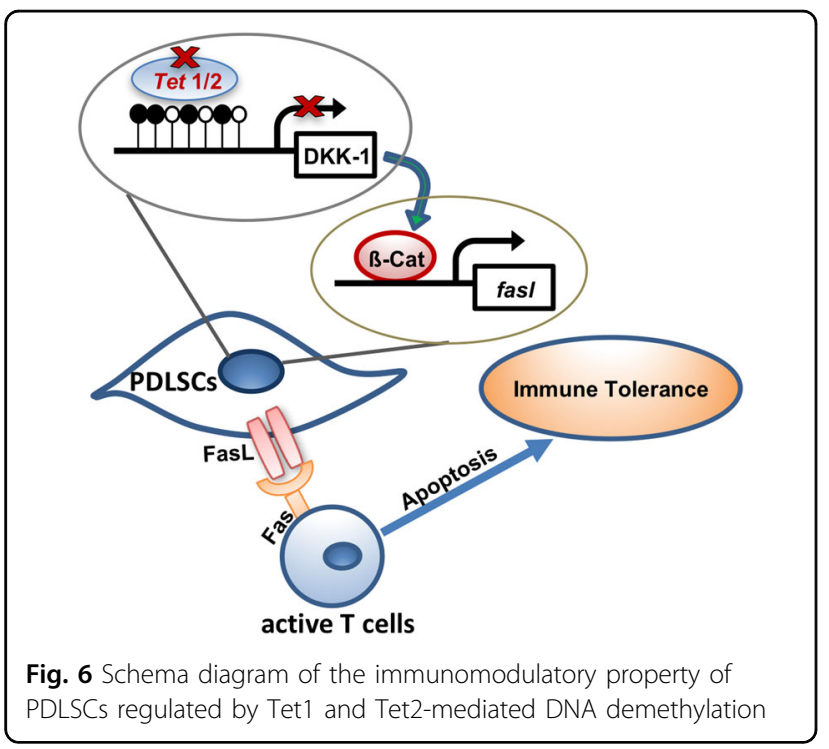

and further promotes FasL expression in PDLSCs to enhance their immunomodulatory ability. This study provided evidence to link Tet1/Tet2, which are DNA demethylases, to MSC-mediated immunomodulation. It has been reported that Tet is able to modulate Foxp3 demethylation in $\mathrm{T}$ cells to affect immune homeostasis ${ }^{10}$ and could directly target the promoters of microRNAs in cancer cells ${ }^{41}$. Except Fas/FasL pathway, we showed that the expression of P-smad3, Notch1, and Notch2 were altered after siRNA knockdown; however, whether PDLSC-mediated immunomodulation could be modulated by other molecules needs further investigation to illustrate the detailed mechanism.

In summary, we demonstrated that Tet1 and Tet2 participate in the regulation of MSC-mediated immunomodulation. Downregulation of Tet1 and Tet2 in PDLSCs improves their immunomodulatory functions by inhibiting $D K K-1$ expression through hypermethylation. Tet1 and Tet2 knockdown pretreatment in PDLSCs may be a promising approach to enhance MSC-mediated immune therapeutic effects.

\section{Materials and Methods Antibodies}

Tet1 (ab191698), Tet2 (ab94580), and CD146 (ab24577) antibodies were purchased from Abcam (Cambridge, MA, USA). Phospho- $\beta$-catenin (Ser552) and $\beta$-catenin (15B8) antibodies were purchased from Invitrogen (Carlsbad, CA, USA). FASL (SC-33716) and DKK-1 (SC-374574) antibodies were purchased from Santa Cruz Biotechnology (Dallas, TX, USA). $\beta$-Actin antibody (A1978) was purchased from Sigma-Aldrich (St. Louis, MO, USA). Anti-CD4-PerCP (550765) and anti-CD25-APC (561048) were purchased from BD Biosciences (San Jose, CA,
USA). Anti-CD3 (100208) and anti-CD28 (102112) were purchased from BioLegend (SanDiego, CA, USA)

\section{Isolation and culture of hPDLSCs}

Periodontal ligament tissues were obtained from healthy patients (18-25 years old) without any history of periodontal disease who were underdoing orthodontic extraction. The protocol of PDLSC isolation and cultivation was followed according to a previous publication by Seo et al. $^{42}$; the procedure was approved by the Ethical Guidelines of Peking University (PKUSSIRB-201311103). Passage 3 PDLSCs were used in all experiments.

\section{siRNA and chemical treatment}

For siRNA transfection, PDLSCs were cultured under reduced serum medium (Opti-MEM, Gibco), and Tet1 siRNA (sc-90457), Tet2 siRNA (sc-88934), or control vehicle siRNA (sc-37007) (Santa Cruz Biotechnology) were treated with Lipofectamine reagent (Invitrogen), according to the manufacturer's instructions. For chemical reagent treatments, serum-starved PDLSCs were treated with $10 \mu \mathrm{M} \mathrm{Wnt/ \beta -catenin} \mathrm{inhibitor} \mathrm{(FH535;}$ Cayman Chemical), or human DKK-1 (PeproTech). Treated cells were collected for further experiments.

\section{Western blotting}

Mammalian protein extraction reagent (Thermo, Rockford, IL, USA) was used to lyse total protein. The protein was applied and separated on 4 to $12 \%$ NuPAGE gel (Invitrogen), which were then transferred to nitrocellulose membranes (Millipore, Billerica, MA, USA). Membranes were blocked with $5 \%$ nonfat dry milk and $0.1 \%$ Tween-20 for $1 \mathrm{~h}$, which were then incubated with the primary antibodies at $4{ }^{\circ} \mathrm{C}$ overnight. We used horseradish peroxidase-conjugated secondary antibody (Santa Cruz Biotechnology; 1:10,000) to treat the membranes for $1 \mathrm{~h}$. By using Super Signal West Pico Chemiluminescent Substrate (Thermo) and BioMax film (Kodak, USA), the immuno-reactive proteins were detected.

\section{Real-time PCR}

Total RNA was isolated using miRNeasy Mini Kit (Qiagen, Valencia, CA, USA) according to the manufacturer's instructions. SuperScript III Reverse Transcriptase (RT) Kit (Invitrogen) was used to prepare the complementary DNA. qPCR was performed using SYBR Green Supermix (Bio-Rad, Hercules, CA, USA) and genespecific primers. Detection was performed on a CFX96 Real-Time PCR System (Bio-Rad).

\section{Immunofluorescent staining}

PDLSCs $\left(1 \times 10^{3} /\right.$ well $)$ were cultured in chamber slides (Nunc, Rochester, NY, USA) and then fixed with $4 \%$ 
paraformaldehyde (PFA). The cells were incubated with primary antibodies at $4{ }^{\circ} \mathrm{C}$ overnight, and then treated with Alexa Fluor 568 or Alexa Fluor 488-conjugated secondary antibody (1:200, Invitrogen) for $1 \mathrm{~h}$. Finally, slides were mounted with Vectashield mounting medium containing 4',6-diamidino-2-phenylindole (DAPI) (Vector Laboratories, Burlingame, CA, USA).

\section{T lymphocytes apoptosis assay}

A total of $0.2 \times 10^{6}$ PDLSCs (with or without Tet1/ 2 siRNA treatment) were seeded on a 24-well culture plate (Corning) containing Dulbecco's modified Eagle's medium (Lonza, Basel, Switzerland) with $10 \%$ heatinactivated fetal bovine serum, $10 \mathrm{mM}$ HEPES, $50 \mu \mathrm{M} 2$ mercaptoethanol, $1 \mathrm{mM}$ sodium pyruvate (SigmaAldrich), 1\% non-essential amino acid (Cambrex, East Rutherford, NY, USA), $2 \mathrm{mM}$ L-glutamine, $100 \mathrm{U} / \mathrm{ml}$ penicillin, and $100 \mu \mathrm{g} / \mathrm{ml}$ streptomycin. Activated T cells $\left(1 \times 10^{6} /\right.$ well $)$, which were isolated from mouse spleen, were pre-stimulated with plate-bound anti-CD3 $\varepsilon$ and anti-CD28 (2 $\mu \mathrm{g} / \mathrm{ml}$ each; BD Bioscience) for 2 days, and then loaded directly on PDLSCs. After $24 \mathrm{~h}$ of coculture, CD3 antibody and AnnexinV Apoptosis Detection Kit I (BD Bioscience) were used to detect apoptotic $\mathrm{T}$ cells using a FACS flow cytometer.

\section{Cell proliferation assay}

PDLSCs were stained with BrdU antibody (1:200, Invitrogen) after being incubated with BrdU solution (1:100, Invitrogen) for $12 \mathrm{~h}$, and then Alexa Fluor 568-conjugated secondary antibody were used to stain the cells for $1 \mathrm{~h}$ at room temperature. Finally, slides were mounted with Vectashield mounting medium containing DAPI (Vector Laboratories). The percentage of BrdU-positive cell number in total cell number was calculated. Three independent samples of each experimental group were used for BrdU assay and BrdU-positive and total cell numbers were counted in 10 images per subject.

\section{DSS-induced mouse colitis and treatment with PDLSCs}

Female C57BL/6J (JAX \#000664) mice were purchased from the Jackson Laboratory (Bar Harbor, ME, USA). Acute colitis was induced in 8-week-old C57BL/6J mice with 3\% (w/v) DSS (MP Biochemicals) in the drinking water for 10 days $^{43}$. A total of $2.0 \times 10^{6}$ untreated PDLSCs or Tet1/Tet2 siRNA-treated PDLSCs were intraperitoneally injected into the mice 3 days after initiation of DSS treatment. DAI was scored on a scale of $0-4$ by evaluating the weight loss, stool consistency/diarrhea, and presence of fecal bleeding ${ }^{19,43}$. At day 10, mice were sacrificed by $\mathrm{CO}_{2}$ euthanasia, their colons were fixed with 4\% PFA, and paraffin-embedded sections were prepared for hematoxylin and eosin staining. Histological scores were blindly determined as previously described ${ }^{19}$. All animal experiments were performed under institutionally approved protocols for the use of animal research (University of Pennsylvania IACUC \#805478).

\section{Chromatin immunoprecipitation-qPCR assays}

PDLSCs were cross-linked and used for each immunoprecipitation (IP). ChIP was performed using the Millipore ChIP Kit according to the manufacturer's protocol. Branson sonifier was used to shear the chromatin. For precipitating DNA-protein complexes, Tet1 and Tet2 antibodies were used and non-specific serum immunoglobulin G was used as an isotype control. Percentage input was determined by removing an aliquot of sheared chromatin prior to IP and comparing amplification of this DNA to amplification of the precipitated chromatin. MethPrimer software was used to predict the presence of $\mathrm{CpG}$ islands in the $D K K-1$ promoter.

\section{Hydroxymethylated DNA immunoprecipitation}

IP of 5-hmC was performed using an Active Motif hMeDIP Kit. A Branson sonicator was used to sonicate DNA into short fragments (100 to 1000 base pairs (bp)), after which it was heat denatured at $95^{\circ} \mathrm{C}$ for $10 \mathrm{~min}$. Sonicated DNA $(1 \mu \mathrm{g})$ was immunoprecipitated with $2.5 \mu \mathrm{g}$ of mouse anti-5-hmC antibody (Active Motif, $1 \mu \mathrm{g} / \mu \mathrm{l}$ ) and was incubated overnight at $4{ }^{\circ} \mathrm{C}$. Then, the magnetic beads were added to the DNA-antibody mixture and isolation of immunoprecipitated DNA was performed according to the kit instructions. SYBR ${ }^{\circ}$ Green Supermix (Bio-Rad) on a Bio-Rad CFX96 Real-Time system was used to perform qPCR, as indicated by the manufacturer's protocol. The amount of DNA used in the IP reaction was calculated for the percentage of enrichment.

\section{Methylation-specific PCR and OxBS-sequencing}

OxBS-sequencing was used to assess DKK-1 methylation status. A highly selective chemical oxidizes $5-\mathrm{hmC}$ to 5-formylcytosine $(5 \mathrm{fC})$ and then bisulfite treatment deformylated and deaminated $5 \mathrm{fC}$ to uracil, appears as thymine $(\mathrm{T})$ in sequencing analysis. $5-\mathrm{mC}$ is not determinate and appears as a cytosine $(\mathrm{C})$ in sequencing analysis. Oxidative bisulfite conversion, which is able to produce an accurate readout distinguishing 5 - $\mathrm{mC}$ from 5hmC, was performed using a TrueMethyl ${ }^{\mathrm{TM}}$ Kit (Cambridge Epigenetix, UK), according to the manufacturer's instructions ${ }^{44}$. For methylation-specific PCR, pretreated DNA was amplified with methylation-specific primers for site 1 and site 2 of DKK- 1 and were sequenced as previously reported by us ${ }^{10}$.

\section{Statistical analysis}

All results are presented as the mean and standard deviation (mean $\pm \mathrm{SD}$ ) for $n$ from 3 to 8 . Independent unpaired two-tailed Student's $t$ tests were used for 
comparing between two groups. One-way analysis of variance was used for comparing between more than two groups. $P$ values $<0.05$ were considered statistically significant.

\section{Acknowledgements}

This work was supported by Beijing Natural Science Foundation No. 7182182 to R.Y.; Peking University Medicine Fund of Fostering Young Scholars' Scientific and Technological Innovation (BMU2018PYB009) to T.Y., supported by "the Fundamental Research Funds for the Central Universities"; the Young Elite Scientist Sponsorship Program by CAST (YESS), 2017QNRC001; the National Natural Science Foundation of China No. 81600865 (R.Y.), 81970940 (R.Y.), 51903003 (T.Y.), and the National Science and Technology Major Project of the Ministry of Science and Technology of China No. 2018ZX10302207.

\section{Authors' contributions}

T.Y. and R.Y. designed the experiments and wrote the manuscript. T.Y., D.L. and T.Z. performed the experiments and collected and analyzed the data. Y.Z. and S.S. designed and revised the manuscript. All co-authors approved the final version of the manuscript for publication.

\section{Author details}

'Department of Orthodontics, Peking University School and Hospital of Stomatology, \#22 Zhongguancun South Avenue, 100081 Beijing, China. ${ }^{2}$ Department of Anatomy and Cell Biology, School of Dental Medicine, University of Pennsylvania, Philadelphia, PA 19104, USA. ${ }^{3}$ South China Center of Craniofacial Stem Cell Research, School of Guanghua Dental Medicine, Sun Yatsen University, \#74 Zhongshan 2 Road, Guangzhou, Guangdong 510080, China

\section{Conflict of interest}

The authors declare that they have no conflict of interest.

\section{Publisher's note}

Springer Nature remains neutral with regard to jurisdictional claims in published maps and institutional affiliations.

Supplementary Information accompanies this paper at (https://doi.org/ 10.1038/s41419-019-2025-z).

Received: 5 June 2019 Revised: 22 August 2019 Accepted: 23 September 2019

Published online: 14 October 2019

\section{References}

1. Ito, S. et al. Role of Tet proteins in $5 \mathrm{mC}$ to $5 \mathrm{hmC}$ conversion, ES-cell selfrenewal and inner cell mass specification. Nature 466, 1129-1133 (2010).

2. Tahiliani, M. et al. Conversion of 5-methylcytosine to 5-hydroxymethylcytosine in mammalian DNA by MLL partner TET1. Science 324, 930-935 (2009).

3. Ito, S. et al. Tet proteins can convert 5-methylcytosine to 5-formylcytosine and 5-carboxylcytosine. Science 333, 1300-1303 (2011).

4. Kim, R., Sheaffer, K. L., Choi, I., Won, K. J. \& Kaestner, K. H. Epigenetic regulation of intestinal stem cells by Tet1-mediated DNA hydroxymethylation. Genes Dev. 30, 2433-2442 (2016).

5. Wu, X., Li, G. \& Xie, R. Decoding the role of TET family dioxygenases in lineage specification. Epigenet. Chromatin 11, 58 (2018).

6. Szwagierczak, A., Bultmann, S., Schmidt, C. S., Spada, F. \& Leonhardt, H. Sensitive enzymatic quantification of 5-hydroxymethylcytosine in genomic DNA. Nucleic Acids Res. 38, e181 (2010).

7. Koh, K. P. et al. Tet1 and Tet2 regulate 5-hydroxymethylcytosine production and cell lineage specification in mouse embryonic stem cells. Cell Stem Cell $\mathbf{8}$, 200-213 (2011).

8. Tan, L. \& Shi, Y. G. Tet family proteins and 5-hydroxymethylcytosine in development and disease. Development 139, 1895-1902 (2012).

9. Yang, R. et al. Tet1 and Tet2 maintain mesenchymal stem cell homeostasis via demethylation of the P2rX7 promoter. Nat. Commun. 9, 2143 (2018).
10. Yang, R. et al. Hydrogen sulfide promotes Tet1- and Tet2-mediated Foxp3 demethylation to drive regulatory $T$ cell differentiation and maintain immune homeostasis. Immunity 43, 251-263 (2015).

11. Whiting, D., Chung, W. O., Johnson, J. D. \& Paranjpe, A. Characterization of the cellular responses of dental mesenchymal stem cells to the immune system. J. Endod. 44, 1126-1131 (2018).

12. Shin, C. et al. Human periodontal ligament stem cells suppress T-cell proliferation via down-regulation of non-classical major histocompatibility complex-like glycoprotein CD1b on dendritic cells. J. Periodontal Res. 52, 135-146 (2017).

13. Liu, D. et al. Mesenchymal stem cells derived from inflamed periodontal ligaments exhibit impaired immunomodulation. J. Clin. Periodontol. 39, 1174-1182 (2012).

14. Liu, $Y$. et al. Periodontal ligament stem cell-mediated treatment for periodontitis in miniature swine. Stem Cells 26, 1065-1073 (2008).

15. Zhai, Q., Dong, Z., Wang, W., Li, B. \& Jin, Y. Dental stem cell and dental tissue regeneration. Front. Med. 13, 152-159 (2019).

16. Guo, L. et al. D-Mannose enhanced immunomodulation of periodontal ligament stem cells via inhibiting IL-6 secretion. Stem Cells Int. 2018, 7168231 (2018).

17. Chen, C. et al. Mesenchymal stem cell transplantation in tight-skin mice identifies miR-151-5p as a therapeutic target for systemic sclerosis. Cell Res. 27, 559-577 (2017).

18. Shi, B. et al. Mesenchymal stem cell transplantation ameliorates Sjogren's syndrome via suppressing IL-12 production by dendritic cells. Stem Cell Res. Ther. 9, 308 (2018).

19. Alex, P. et al. Distinct cytokine patterns identified from multiplex profiles of murine DSS and TNBS-induced colitis. Inflamm. Bowel Dis. 15, 341-352 (2009).

20. Akiyama, K. et al. Mesenchymal-stem-cell-induced immunoregulation involves FAS-ligand-/FAS-mediated T cell apoptosis. Cell Stem Cell 10, 544-555 (2012).

21. Kohli, R. M. \& Zhang, Y. TET enzymes, TDG and the dynamics of DNA demethylation. Nature 502, 472-479 (2013).

22. $\mathrm{Xu}$, Y. et al. Genome-wide regulation of $5 \mathrm{hmC}, 5 \mathrm{mC}$, and gene expression by Tet1 hydroxylase in mouse embryonic stem cells. Mol. Cell 42, 451-464 (2011).

23. Chen, $\mathrm{C}$. et al. Telomerase governs immunomodulatory properties of mesenchymal stem cells by regulating FAS ligand expression. EMBO Mol. Med. 6, 322-334 (2014).

24. Liu, Y. et al. Acetylsalicylic acid treatment improves differentiation and immunomodulation of SHED. J. Dent. Res. 94, 209-218 (2015).

25. Lin, G. L. \& Hankenson, K. D. Integration of BMP, Wnt, and notch signaling pathways in osteoblast differentiation. J. Cell Biochem. 112, 3491-3501 (2011).

26. Luo, K. Signaling cross talk between TGF-beta/Smad and other signaling pathways. Cold Spring Harb. Perspect. Biol. 9, a022137 (2017).

27. Guo, Y. et al. The Wnt inhibitor Dkk1 is required for maintaining the normal cardiac differentiation program in Xenopus laevis. Dev. Biol. 449, 1-13 (2019).

28. Nakatsukasa, H. et al. Loss of TET proteins in regulatory $T$ cells promotes abnormal proliferation, Foxp3 destabilization, and IL-17 expression. Int. Immunol. 31, 335-347 (2019).

29. Lio, C. J. \& Rao, A. TET Enzymes and $5 \mathrm{hmC}$ in adaptive and innate immune systems. Front. Immunol. 10, 210 (2019).

30. An, J. et al. Acute loss of TET function results in aggressive myeloid cancer in mice. Nat. Commun. 6, 10071 (2015).

31. Huang, Y. \& Rao, A. Connections between TET proteins and aberrant DNA modification in cancer. Trends Genet. 30, 464-474 (2014).

32. Tsagaratou, A. et al. TET proteins regulate the lineage specification and TCRmediated expansion of iNKT cells. Nat. Immunol. 18, 45-53 (2017).

33. Li, Q. et al. FAM2OC could be targeted by TET1 to promote odontoblastic differentiation potential of human dental pulp cells. Cell Prolif. 51, e12426 (2018).

34. Mahaira, L. G. et al. IGF2BP1 expression in human mesenchymal stem cells significantly affects their proliferation and is under the epigenetic control of TET1/2 demethylases. Stem Cells Dev. 23, 2501-2512 (2014).

35. Sajadian, S. O. et al. Vitamin C enhances epigenetic modifications induced by 5 -azacytidine and cell cycle arrest in the hepatocellular carcinoma cell lines HLE and Huh7. Clin. Epigenet. 8, 46 (2016).

36. Squillaro, T., Peluso, G. \& Galderisi, U. Clinical trials with mesenchymal stem cells: an update. Cell Transplant. 25, 829-848 (2016).

37. Samsonraj, R. M. et al. Concise review: multifaceted characterization of human mesenchymal stem cells for use in regenerative medicine. Stem Cells Transl. Med. 6, 2173-2185 (2017). 
38. Aguilera, O. et al. Epigenetic inactivation of the Wnt antagonist DICKKOPF-1 (DKK-1) gene in human colorectal cancer. Oncogene 25, 4116-4121 (2006).

39. Pannone, G. et al. WNT pathway in oral cancer: epigenetic inactivation of WNT-inhibitors. Oncol. Rep. 24, 1035-1041 (2010)

40. Neri, F. et al. TET1 is a tumour suppressor that inhibits colon cancer growth by derepressing inhibitors of the WNT pathway. Oncogene 34, 4168-4176 (2015)

41. Atlante, S. et al. Alpha-ketoglutarate dehydrogenase inhibition counteracts breast cancer-associated lung metastasis. Cell Death Dis. 9, 756 (2018).
42. Seo, B. M. et al. Investigation of multipotent postnatal stem cells from human periodontal ligament. Lancet 364, 149-155 (2004).

43. Zhang, Q. et al. Mesenchymal stem cells derived from human gingiva are capable of immunomodulatory functions and ameliorate inflammationrelated tissue destruction in experimental colitis. J. Immunol. 183, 7787-7798 (2009).

44. Booth, M. J. et al. Oxidative bisulfite sequencing of 5-methylcytosine and 5hydroxymethylcytosine. Nat. Protoc. 8, 1841-1851 (2013). 